\title{
Simplifying Electron Beam Channeling in STEM
}

\author{
Ryan J. Wu, Anudha Mittal, Michael L. Odlyzko, K. Andre Mkhoyan \\ Department of Chemical Engineering and Materials Science, University of Minnesota, MN, USA
}

The channeling behavior for electron probes in conventional transmission electron microscopy (TEM) and scanning TEM (STEM) remains an active area of research because it influences the quantitative interpretation of high-resolution images and spectroscopies. Electron beam channeling causes oscillations in the STEM probe intensity during specimen propagation, which leads to differences in the number of electrons incident at different depths [1]. Understanding these short-range oscillations and the parameters that influence them is critical due to the short depths of focus of probes in modern aberration-corrected STEMs. Although sophisticated mathematical descriptions have modeled beam channeling accurately [2, 3], a less rigorous approach can provide a more accessible understanding of how this complex phenomenon affects STEM results acquired from experiments.

In this work, we attempt to simplify and generalize the description of electron beam channeling's origins, behavior, and governing parameters in order to bridge the gap between theoretical models and experimental applications. Multislice simulations [4] are performed to visualize electron channeling through beam intensity depth profiles in real and reciprocal space (Figure 1). These profiles provide a simple description of beam channeling as one governed by the initial angular distribution and redistribution of the beam during sample propagation.

Furthermore, the STEM probe parameters and sample characteristics are varied to explore their effects on channeling behavior. Figure 2 shows three contrasting regimes of electron channeling as a function of $Z$ : the high-atomic-number $(Z)$ regime, in which intensity oscillations during channeling occur only along single atomic columns and scattering leads to significant angular redistribution of the beam; the low- $Z$ regime, in which beam intensity shifts to neighboring columns; and the initial angular distribution of the probe controls intensity oscillations; and the intermediate $\mathrm{Z}$ regime which shows mixed beam characteristic. These regimes are further generalized by a simple mathematical description that relates the probe parameters (convergence angle and energy of the beam) and the sample characteristics ( $Z$ and crystal lattice parameters) to predict the channeling behavior of the electron beam during experiment.

Finally, this description of channeling is applied to polyatomic crystals with irregular spacing between atoms to simulate a more general experimental scenario. Results show that the electron beam channels as if the parameters of the crystal are averaged over the entire sample as oppose to parameters at each scattering atom. These results provide a new understanding of the occurrence and consequences of channeling phenomena, as well as conditions under which their influence is strengthened or weakened by characteristics of the electron probe and sample [5].

[1] L. F. Kourkoutis et al., Physical Review B 84, (2011) 075485

[2] M. Op de Beeck \& D. Van Dyck, Ultramicroscopy 64, (1996), pg 153-165.

[3] S. J. Pennycook, Ultramicroscopy 26, (1988), pg 239-248. 
[4] E. J. Kirkland, Advanced Computing in Electron Microscopy, 2nd ed. New York, 2010.

[5] The authors gratefully acknowledge funding provided by C-SPIN, one of six centers of STARnet, a Semiconductor Research Corporation program, sponsored by MARCO and DARPA.
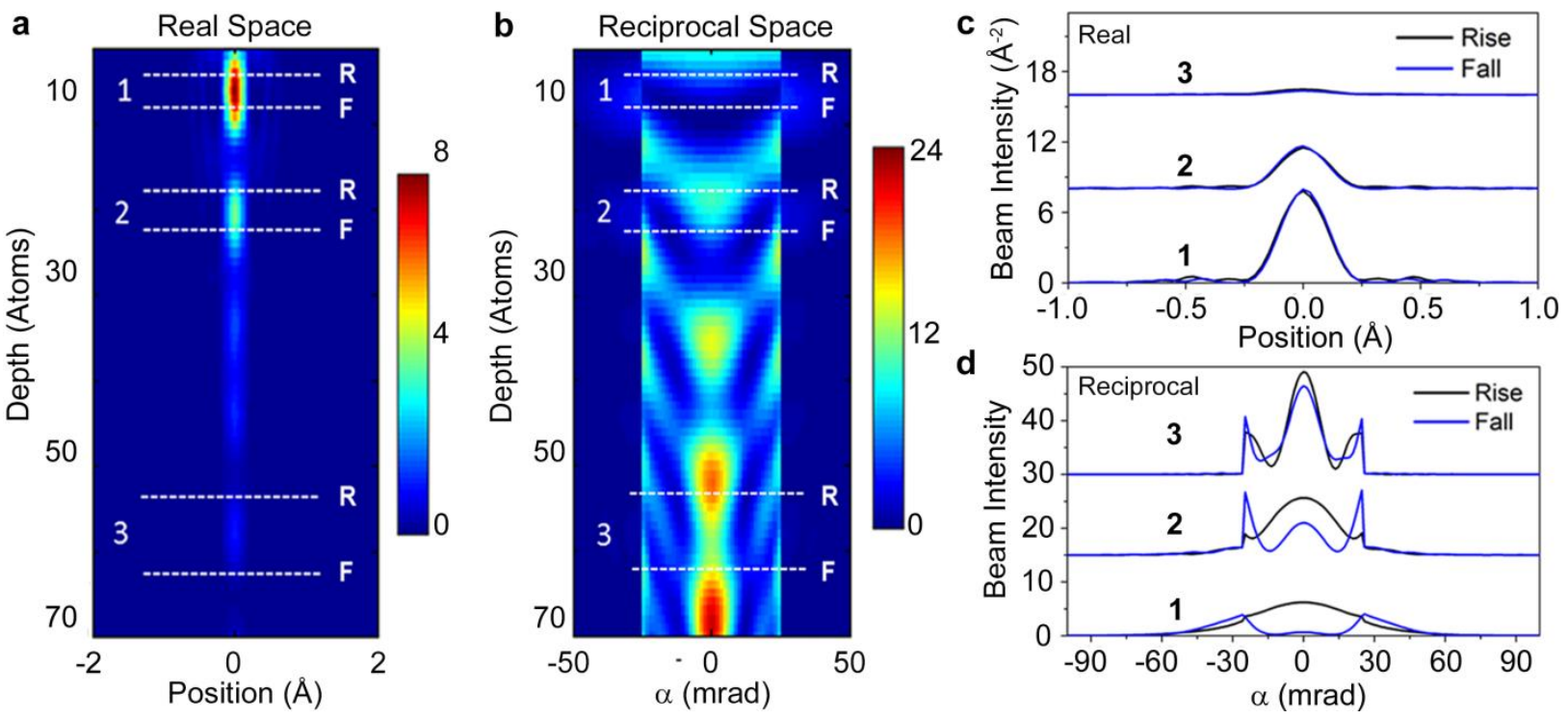

Figure 1: (a) 2D beam intensity depth profile of a STEM probe propagating through a Ga column. Depth locations of equal intensities are indicated with dashed lines and distinguished to show whether the beam intensity is rising or falling at that location. (b) 2D beam intensity depth profile of the same STEM probe in reciprocal (or angular) space $(k=\alpha / \lambda)$. (c) Line scans of spatial distributions at different depths corresponding to pairs of position marked by dashed lines in (a). (d) Line scans of angular distributions corresponding to positions marked by dashed lines in (b).
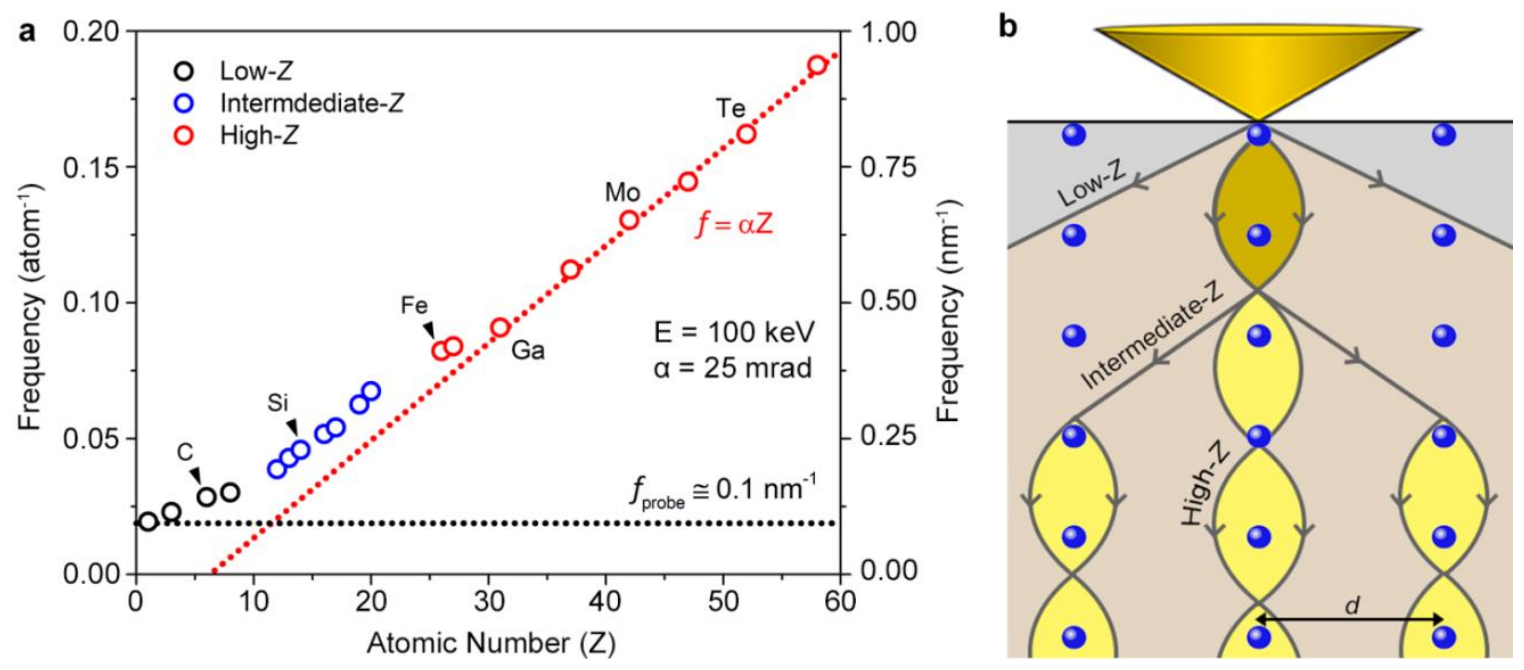

Figure 2: (a) Frequency of oscillation in beam intensity along the optic axis in atomic columns of different elements. The dotted black line represents the frequency of oscillation in vacuum without an element. The red dotted line is a linear fit, $f=a Z$, of data points between $Z=40$ to $Z=60$ that are representative of very high- $Z$ elements. (b) Schematic diagram showing the paths of the beam intensity from the STEM probe as it propagates in low-, intermediate- and high- $Z$ crystals. 